\title{
EDITORIAL
}

\section{Depression, stress, and the heart}

\section{P C Strike, A Steptoe}

Evidence is accumulating that psychosocial factors, in particular depression, contribute to coronary artery disease

See end of article for authors' affiliations

......................

Correspondence to: Dr PC Strike, Department of Epidemiology and Public Health, University College London, 1-19 Torrington Place, London WC1E 6BT, UK; philip@

public-health.ucl.ac.uk
$\mathrm{T}$ here is rapidly growing interest in the interactions between heart and mind. Evidence is accumulating from epidemiological and clinical studies that psychosocial factors contribute to coronary artery disease (CAD). Depression is a common problem with a high degree of associated disability, and has been the focus of much of this work. Depressed individuals are more likely to develop angina, or fatal or non-fatal myocardial infarction, than their non-depressed counterparts. A review of the prospective epidemiological cohort studies into depression and the subsequent development of CAD has shown a possible aetiologic role for depression or hopelessness in eight out of eight studies, even after adjustment for traditional cardiovascular risk factors. ${ }^{1}$ Overall these studies show a relative risk of approximately twofold of developing $\mathrm{CAD}$ for depressed compared with non-depressed groups, with some studies showing a dose-response effect in which greater exposure to depression leads to a higher incidence of coronary events. ${ }^{2}$ For example, a four year follow up of the Amsterdam longitudinal ageing study reported a relative risk of cardiac mortality of 1.6 for individuals with depressive symptoms, and 3.8 for those with clinically diagnosed depression, after adjustment for age, sex, education, smoking, alcohol, hypertension, body mass index, diabetes, stroke, and cancer. ${ }^{2}$

The John Hopkins precursors study $y^{3}$ followed 1190 medical students for a median of 37 years, and found a median interval of 15 years between the first episode of depression and the first coronary event. This suggests a chronic underlying link, with depression pre-dating the development of clinical CAD. Duration of symptoms themselves may also influence the risk of mortality. ${ }^{4}$ More recently, it has been found in the Whitehall II study that British civil servants with elevated scores on the general health questionnaire (an inventory screening for psychological distress in the general population) were at increased risk of developing coronary heart disease over a five year period, independently of other psychosocial factors including socioeconomic status. ${ }^{5}$

\section{DEPRESSION FOLLOWING MYOCARDIAL INFARCTION}

Depression is an adverse prognostic factor in patients with a diagnosis of $\mathrm{CAD}^{1}{ }^{1}$ Between $15-20 \%$ of patients experience major depressive illness after myocardial infarction, and many more experience depressive symptoms. There is a threefold greater incidence of depression in CAD patients compared with the general public. ${ }^{1}$ The majority of studies suggest an association with poor prognosis, ${ }^{46}$ although this has not been a uniform finding. ${ }^{7}$ Recently, a link between level of depressive symptoms assessed soon after myocardial infarction and five year survival has been demonstrated. ${ }^{6}$ These findings have prompted a search for the psychobiological mechanisms linking CAD and depression.

\section{IMMUNOLOGICAL ABNORMALITIES}

Depression is associated with abnormalities of platelet function, blood coagulation, immune function, the hypothalamic-pituitary-adrenocortical axis, autonomic balance, folate/homocysteine metabolism, and endothelial function, all of which may play a role in the atherosclerotic disease process. With the growing evidence for atherosclerosis as an inflammatory process, there has been much interest in inflammation as a mediator between depression and CAD. Depression is associated with an acute phase response as evidenced by a higher $\mathrm{C}$ reactive protein and raised concentrations of pro-inflammatory cytokines such as interleukin-6 (IL-6). Interleukin-1 $\beta$ (IL-1 $\beta)$, tumour necrosis factor $\alpha(\mathrm{TNF} \alpha)$ as well as interleukin-1 receptor antagonist (IL-1Ra) are also raised in depressed patients. ${ }^{89}$ These proinflammatory cytokines can produce symptoms of depression, anorexia, weight loss, malaise, anhedonia, and sleep disturbances. ${ }^{810}$ Raised concentrations of intercellular adhesion molecule-1 (ICAM-1), E-selectin, and monocyte chemoattractant protein-1 (MCP-1) are present in depressed individuals as well, ${ }^{11}$ and may play a role in the cellular infiltration associated with atherosclerotic disease.

\section{HAEMATOLOGICAL ABNORMALITIES}

Depressed persons also have haematological abnormalities leading to a prothrombotic state. There is a higher white blood cell count, fibrinogen, and factor VIIc in depressed than non-depressed people. ${ }^{12}$ Concentrations of platelet factor IV and $\beta$ thromboglobulin are also raised. ${ }^{1}$ The raised IL-6 in depression is positively correlated with the number of leucocytes, neutrophils, and $\mathrm{CD} 2+\mathrm{T}$ and $\mathrm{CD} 4+\mathrm{T}$ cells ${ }^{8}$. Platelet activation may contribute to the link between depression, thrombus formation, and haemostasis. Platelet activation is greater in depressed than

Abbreviations: $C A D$, coronary artery disease; FMD, flow mediated dilatation; HT, hydroxytryptamine; ICAM-1, intercellular adhesion molecule-1; IL, interleukin; MCP-1, monocyte chemoattractant protein-1; TNF $\alpha$, tumour necrosis factor $\alpha$ 
non-depressed patients with CAD, with increased activation of glycoprotein IIb/IIIa receptors. ${ }^{12}{ }^{13}$ Depressed patients without CAD also have significantly increased 5 hydroxytryptamine (HT) mediated platelet activity compared with non-depressed controls, possibly caused by upregulation of platelet $5 \mathrm{HT}_{2 \mathrm{~A}}$ receptors. ${ }^{14}$

The association between depression and CAD may be explained in several possible ways: depression could lead to inflammation which in turn leads to CAD; an inflammatory process could cause both central nervous system disturbances (and depression) and CAD; both could be a product of atherosclerotic vascular disease; both could arise from chronic strain in the social environment; or depression could lead to CAD via the adoption of health compromising behaviours such as smoking, poor diet, lack of exercise, and poor adherence to treatment.

\section{VASCULAR ENDOTHELIAL DYSFUNCTION}

The study by Broadley and colleagues in this issue of Heart points to another pathophysiological process relating depression with cardiovascular risk, namely vascular endothelial dysfunction. ${ }^{15}$ Endothelium dependent flow mediated dilatation (FMD) was assessed in the brachial arteries of patients with proven depression currently taking antidepressant therapy, and compared with that of non-depressed controls. The depressed patients showed impaired FMD. Patients had been treated for a minimum of three months, were considered to be in remission at the time of study, and none had pronounced cardiovascular risk factors. These results are complimentary to the work described by Rajagopalan and colleagues, ${ }^{11}$ who demonstrated a pronounced attenuation of FMD in young depressed patients without conventional cardiovascular risk factors who were not taking antidepressant treatment. This strongly suggests that the abnormality of endothelial function is not a side effect of medication or secondary to traditional risk factors.

Rajagopalan and colleagues found a 3.8\% increase in brachial artery diameter in their depressed patients compared with a $9.6 \%$ increase in the control group. The percentage changes in FMD seen in Broadley's study were $-0.7 \%$ in the depressed patients versus $5.7 \%$ in the control group. The glyceryl trinitrate induced endothelium independent dilatation was very similar in the two studies and was similar to controls. The patients in Broadley's study were 10 years older on average than those in Rajagopalan's study, and this, or indeed the presence of antidepressant medication, may have contributed to the difference in absolute FMD response. No information is given regarding duration of depressive symptoms. Endothelial function is significantly affected by cardiovascular risk factors such as smoking, hypertension, and lack of physical fitness. All are typically more prevalent in depressed persons in the community, which may mean that the degree of endothelial impairment seen in these studies is an underestimate of the true effects.

Brief episodes of acute mental stress have been shown to cause endothelial dysfunction for up to 1.5 hours in healthy individuals. ${ }^{16}$ Nitric oxide production has an antiatherogenic effect by inhibiting cellular adhesion, migration, and proliferative responses, so stress related endothelial impairment may affect the integrity of the vascular endothelium. If stress responses are chronic or repeatedly elicited during everyday life, the result may be acceleration of atherogenesis. ${ }^{16}$ One model of depression is that it mirrors a low grade chronic emotional stress reaction. This might lead to subclinical disturbances of the endothelial, haematological, immune, and neuroendocrine function, allowing the initiation and subsequent progression of atherosclerotic lesions. The psychosocial factors, apart from depression, that have been most consistently associated in prospective studies with CAD are low socioeconomic status, work related stress, and social isolation. ${ }^{1}$ These factors may cluster with depressive symptoms, further exacerbating risk.

\section{ANTIDEPRESSANT TREATMENT}

Depression can be successfully symptomatically treated in depressed patients with CAD. ${ }^{17}$ However, there has been concern that some medications such as tricyclic antidepressants have a cardiotoxic effect ${ }^{17}$ on rate and rhythm. There is also evidence that selective serotonin reuptake inhibitors, especially paroxitine, have in vitro and in vivo inhibitory effects on nitric oxide synthase, and this may translate into a deleterious effect on endothelial function. This clearly has implications for its use in patients with CAD, although more evidence is needed to change current practice. Pharmacological treatment is unlikely to be appropriate for all patients with $\mathrm{CAD}$, since cardiac risk is apparently increased at subclinical levels of depressive symptoms for which drug treatment is not warranted. However, initial reports from recent trials of cognitive behaviour therapy (ENRICHD-enhancing recovery in coronary heart disease patients) and sertraline (SADHART sertraline antidepressant heart attack randomised trial) for depressed patients following myocardial infarction have also yielded disappointing results. ${ }^{18}$

It is interesting that Broadley and colleagues ${ }^{15}$ observed impaired endothelial function, even though depression had been effectively treated. Other groups have also found that treating depression does not reverse all of the accompanying pathophysiological abnormalities. Maes and associates ${ }^{8}$ found that antidepressant treatment did not significantly reduce the raised serum IL- 6 or IL-1 Ra concentrations in depressed patients, although the elevation in IL- 6 was more pronounced in the patients with treatment resistant depression. Similarly, effective treatment is not associated with normalisation of the abnormalities of heart rate variability ${ }^{17}$ or baroreflex sensitivity ${ }^{15}$ seen in depression. It is probable that treatment for depression will not reduce mortality and morbidity from CAD unless it tackles the underlying link between depression and CAD, rather than just the symptoms of depression. Medication affecting the symptoms alone may have limited benefits which need to be balanced against deleterious effects. It may be that some depression in cardiac patients is a manifestation of an underlying inflammatory process, and that treating the symptoms does not necessarily treat the crucial elements of the disease.

\section{Authors' affiliation}

P C Strike, A Steptoe, Department of Epidemiology and Public Health, University College London, London, UK

\section{REFERENCES}

1 Rozanski A, Blumenthal JA, Kaplan J. Impact of psychological factors on the pathogenesis of cardiovascular disease and implications for therapy. Circulation 1999:99:2192-217.

2 Penninx BW, Beekman AT, Honig A, et al. Depression and cardiac mortality: results from a community-based longitudinal study. Arch Gen Psychiatry 2001;58:221-7.

3 Ford DE, Mead LA, Chang PP, et al. Depression is a risk factor for coronary artery disease in men: the precursors study. Arch Intern Med 1998; 158: 1422-6.

4 Stansfeld S, Fuhrer R. Depression and coronary heart disease. In: Stansfeld S, Marmot M, eds. Stress and the heart. London: BM Books, 2002:101-23.

5 Stansfeld SA, Fuhrer R, Shipley M, et al. Psychological distress as a risk factor for coronary heart disease in the Whitehall II study. Int J Epidemiol 2002;31:248-55

6 Lesperance F, Frasure-Smith N, Talajic M, et al. Five-year risk of cardiac mortality in relation to initial severity and one-year changes in depression symptoms after myocardial infarction. Circulation 2002;105:1049-53.

7 Lane D, Carroll D, Ring C, et al. Mortality and quality of life 12 months after myocardial infarction: effects of depression and anxiety. Psychosom Med 2001;63:221-30.

8 Maes M, Bosmans E, De Jongh R, et al. Increased serum IL-6 and IL-1 receptor antagonist concentrations in major depression and treatment resistant depression. Cytokine 1997;9:853-8

9 Appels A, Bar FW, Bar J, et al. Inflammation, depressive symptomtology, and coronary artery disease. Psychosom Med 2000;62:601-5.

10 Maes M. Major depression and activation of the inflammatory response system. Adv Exp Med Biol 1999;461:25-46. 
11 Rajagopalan S, Brook R, Rubenfire $M$, et al. Abnormal brachial artery flow-mediated vasodilation in young adults with major depression. Am J Cardiol 2001;88:196-8,A7

12 Kop WJ, Gottdiener JS, Tangen CM, et al. Inflammation and coagulation factors in persons $>65$ years of age with symptoms of depression but without evidence of myocardial ischemia. Am J Cardiol 2002;89:419-24.

13 Nemeroff CB, Musselman DL. Are platelets the link between depression and ischemic heart disease? Am Heart J 2000;140:57-62.

14 Shimbo D, Child J, Davidson K, et al. Exaggerated serotonin-mediated platelet reactivity as a possible link in depression and acute coronary syndromes. Am J Cardiol 2002;89:331-3.

15 Broadley AJM, Korszun A, Jones $\mathrm{CJH}$, et al. Arterial endothelial function is impaired in treated depression. Heart 2002:88:521-4.
16 Ghiadoni L, Donald AE, Cropley M, et al. Mental stress induces transient endothelial dysfunction in humans. Circulation 2000; 102:2473-8

17 Carney RM, Freedland KE, Veith RC, et al. Can treating depression reduce mortality after an acute myocardial infarction? Psychosom Med $1999 \cdot 61 \cdot 666-75$

18 Louis AA, Manousos IR, Coletta AP, et al. Clinical trials update: The heart protection study, IONA, CARISA, ENRICHD, ACUTE, ALIVE, MADIT II and REMATCH. Impact of nicorandil on angina. Combination assessment of ranolazine in stable angina. Enhancing recovery in coronary heart disease patients. Assessment of cardioversion using transoesophageal echocardiography. Azimilide post-infarct survival evaluation. Randomised evaluation of mechanical assistance for treatment of chronic heart failure. Eur J Heart Failure 2002; 4:111-6.

\section{IMAGES IN CARDIOLOGY}

\section{Ventricular tachycardia as a presenting feature of dermatomyositis}

A 47 year old man suffered recurrent episodes of exertional syncope for three months, and hospital admission was precipitated by syncope while driving. A telemetry recording showed episodes of ventricular tachycardia during minimal exertion (below left). He was noted to have Gottron's sign (fuscia coloured, elevated, slightly scaly patches over the knuckles indicating dermatomyositis) (right, upper panel) and "car mechanic's" hands (fissuring of the paraungual areas seen in dermatomyositis and overlap connective tissue disease) (lower panel), and reported proximal muscle pain. Further investigation revealed normal coronary arteries, and a persistently elevated troponin I concentration, consistent with the clinical diagnosis of dermatomyositis complicated by cardiac muscle involvement. Computed tomographic scan of the thorax confirmed the presence of pulmonary fibrosis as a feature of his connective tissue disease.

Treatment with sotalol $40 \mathrm{mg}$ three times daily, prednisolone $40 \mathrm{mg}$ once a day, and azathioprine $150 \mathrm{mg}$ once a day led to resolution of symptoms and a decrease in troponin I concentrations to the normal range. After 18 months of follow up the patient remains well and free of syncope. This case illustrates the importance of cardiac muscle involvement in dermatomyositis. Troponin I but not creatine kinase

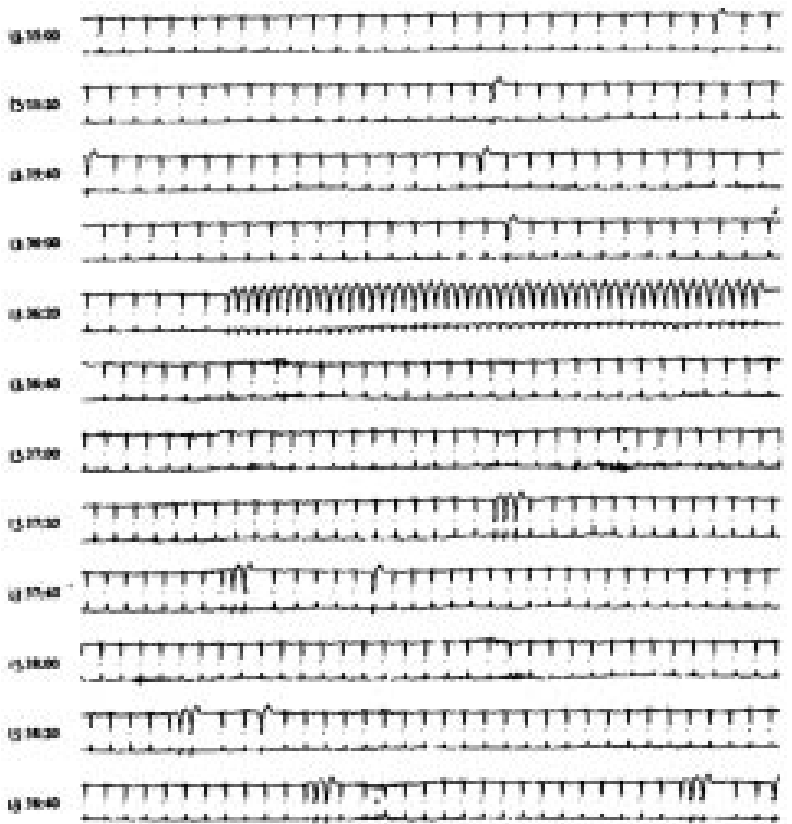

$\mathrm{MB}$, which is overexpressed by regenerating skeletal muscle and usually elevated in dermatomyositis, might be useful to monitor cardiac muscle involvement in this disease.

M Adler

S Banerjeee

R Stratton

rjstratton.18@virgin.net
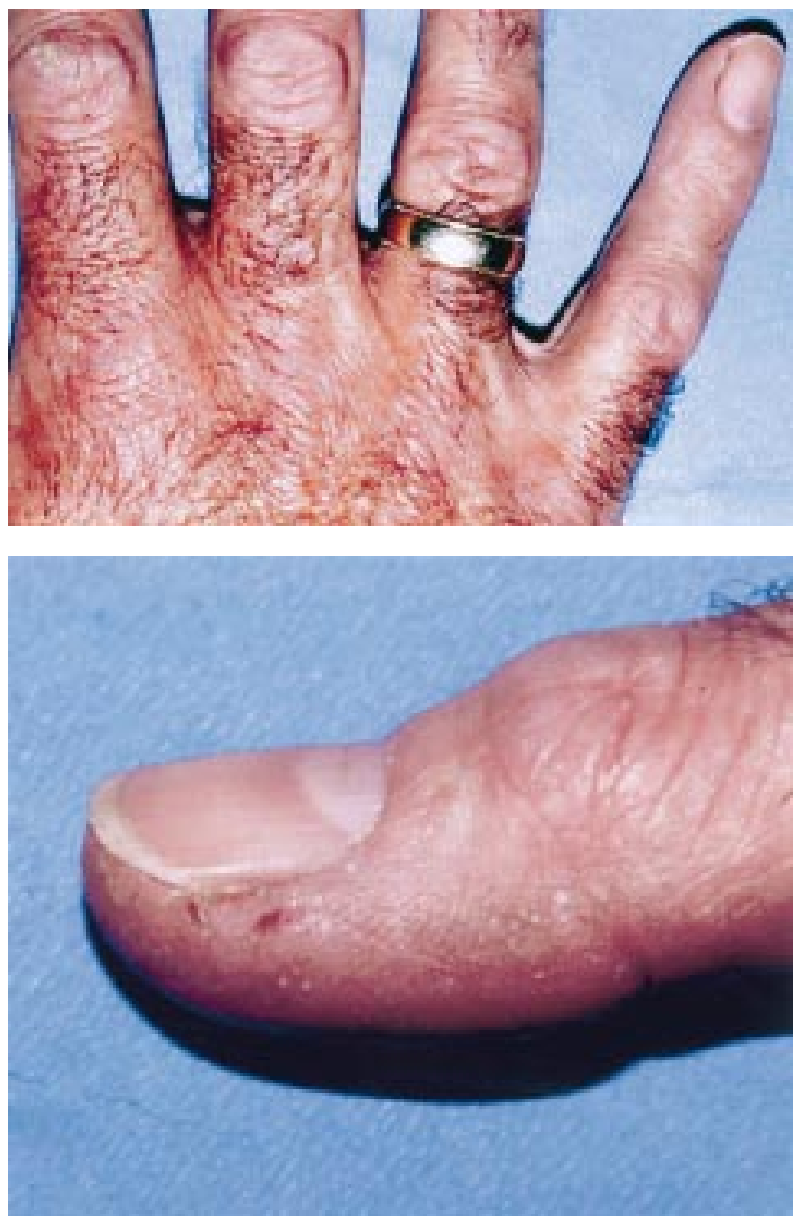